\title{
Iron deficiency anemia (ida) and iron deficiency (id): are alginates a good choise to improve oral iron bioavailability and safety?
}

\author{
Mara Baldacci, Giovanni Battista Gervasi and Marco Bertini* \\ R\&D Department, Laboratori Baldacci SpA, Via San Michele Degli Scalzi 73, Italy
}

\section{Introduction}

One-half of anemia cases are related to iron deficiency (ID) and iron deficiency anemia (IDA) represent also in 2017 the most common nutritional disorders worldwide [1].

Oral iron still remains the first-line therapy for treating ID and IDA but, unfortunately, this cost-effective and convenient treatment, presents gastrointestinal adverse effects such as nausea, diarrhea, epigastric discomfort and costipation that usually decrease the adherence of patients to domiciliary compliance protocol [2-4].

These adverse effects may be reduced by meals but absorption of elemental iron may dcrease by $40 \%$ by food [2-4].

Oral Ferrous $(\mathrm{Fe}++)$ iron formulations are recommended by the International Nutritional Anemia Consultive Group (INACG), from the World Health Organization (WHO) and from the United Nations Children's Fund (UNICEF) for ID and IDA treatment instead of Ferric iron $(\mathrm{Fe}+++)$ because the latter is less available when administered by mouth [2-4].

So that, improve oral iron therapy compliance seems to be "a necessary goal" to reduce ID and IDA prevalence all over the world.

Sodium Alginate (ALG) is a non-toxic, biocompatible, biodegradable polymer, which belongs to the polysaccharides naturally present in seaweed [5-6].

The contact between ALG and the acidic environment in the stomach leads to the formation of a gel layer that possess a protective effect on the mucosa membranes of the stomach and oesophagus: for this reason ALG is an ingedient of many medications (antacids) commonly used in the treatment of hearthburn and reflux diseases $[7,8]$.

Mucoadhesive Microsphere of ALG have recently demostrated to be a unique "carrier system" for many pharmaceuticals preparation increasing oral iron bioavailability of many drugs such as metformin, amoxicillin, furosemide, ibuprofen, insulin, acyclovir, captopril, glipizide and dicumarol [9-11] resulting in a promising drug delivery system [12].

Recently, ALG microsphere with Ranitidine obtained by spraydrying techniques have shown to improve Ranitidine oral iron bioavaiability [12] confirming that ALG co-processed with other substances by spray-drying technique could represent an innovative and cost-effective method to improve oral bioavailability and to reduce gastrointestinal drug's side effects [12].
Zein-Alginate based oral drug delivery system have demostrated to protect Superoxide Dismutase (SOD) from the harsh conditions of gastrointestinal tract [13] and cissus-gum employed as polymer with sodium alginate in the formulation of diclofenac microbeds have shown to reduce gastric side effects of diclofenac in rats [14].

Spray-drying techniques has recently been used to obtained a new co-processed compound between ALG and Ferrous Bysglicinate Chelate named "FERALGINE", in which Sodium Alginate (ALG) and Ferrous Bysglicinate Chelate are present in a 1 to 1 ratio, but without ALG microsphere, and in which every single, little particle of the powder possess the same morphology and quantity of the two different co-processed substances (Figure 1).

Ferrous Bysglicinate Chelate consists of one molecule of Ferrous ion bound to two molecules of glycine to form two heterocyclic rings (Figure 2).

It has been chosen because of its Ferrous form $(\mathrm{Fe}++)$ and because of its particular and interesting pharmacokinetic/pharmacodynamic profile allowing to the compound a safer and more bioavalable oral iron supplementation when confronted with Ferrous Sulphate [15].

Increase oral bioavailability, reduce gastrointestinal adverse events after oral administration and shortner the time of onset of iron replacement therapy versus Ferrous Bysglicinate alone or versus every available form of iron salts have been considered the objectives of this new co-processed compound.

Preliminary clinical trials seems to confirm the "dispert effect" of ALG on Ferrous Bysglicinate Chelate allowing to FERALGINE ${ }^{\mathrm{rn}}$ a more bioavalable and fastner profile in terms of iron replacement if confronted with Ferrous Sulphate after Oral Iron Absorption Test (OIAT) (Figure 3).

Amè and Coll have demostrated an increase of $1.11 \mathrm{~g} / \mathrm{dl}$ of Haemoglobin in patients affected by IDA after 35-60 days of treatment with only $30 \mathrm{mg}$ of elemental iron belonging to FERALGINE ${ }^{\mathrm{m}}$ daily without gastrointestinal adverse events (Figure 4).

Correspondence to: Marco Bertini, R\&D Department, Laboratori Baldacci SpA, Via San Michele Degli Scalzi 73, Italy, E-mail: bertini@baldaccilab.com

Received: November 28, 2017; Accepted: January 11, 2018; Published: January 15,2018 


\section{Conclusion}

Concluding, considering the low quantity of elemental iron (30 $\mathrm{mg}$ /day) administered by oral route able to restore Haemoglobin and the quick increase in Iron blood concentration after OIAT with three/ four less quantity of elemental iron if confronted with the gold standard "Ferrous Sulphate", it seems that ALG could confirm previous results making this 1 to 1 ratio combination between Ferrous Bysglicinate Chelate and Sodium Alginate (ALG) by spray-drying techniques a new interesting co-processed formulation able to improve ID and IDA treatment with a very safe profile in terms of gastrointestinal adverse events.

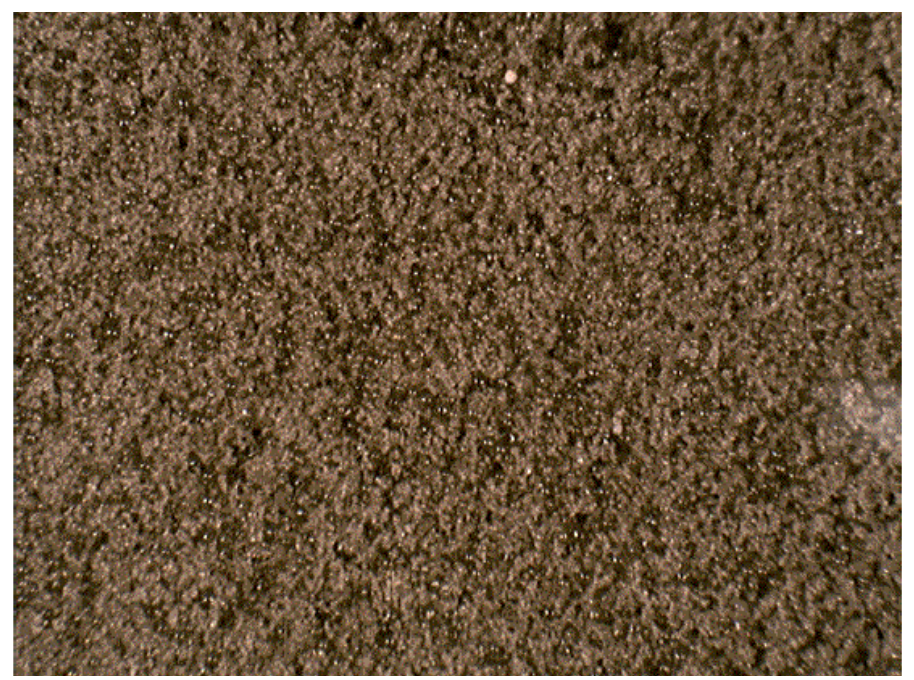

Figure 1. Feralgina powder (picture making by stereomicroscopy Wild Heerbrugg Markroskop M420 linked to an OPTIKAM MICROSCOPY DIGITAL USB CAMERA [3].

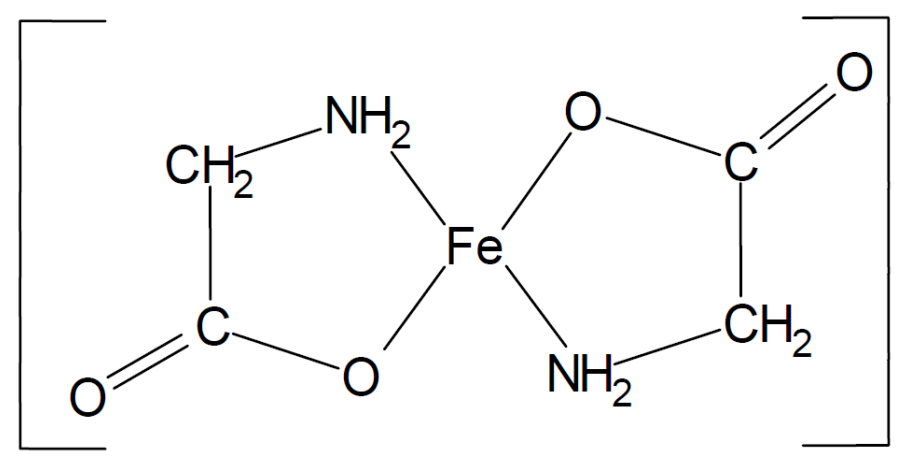

Figure 2. Structural formula (15)

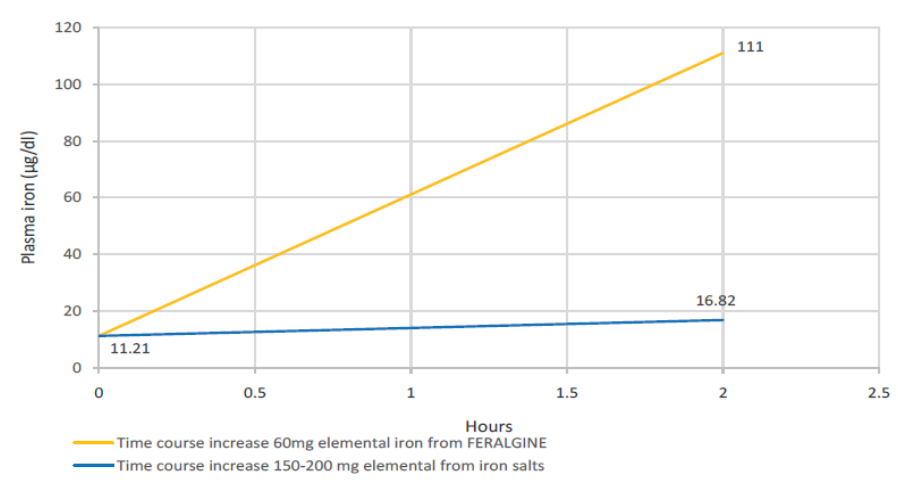

Figure 3. Expected theoretical plasma iron level after 2 hours from $200 \mathrm{mg}$ of elemental iron oral salts administration (blue lines) versus experimental FERALGINE $60 \mathrm{mg}$ elemental iron oral administration (yellow line) (4).

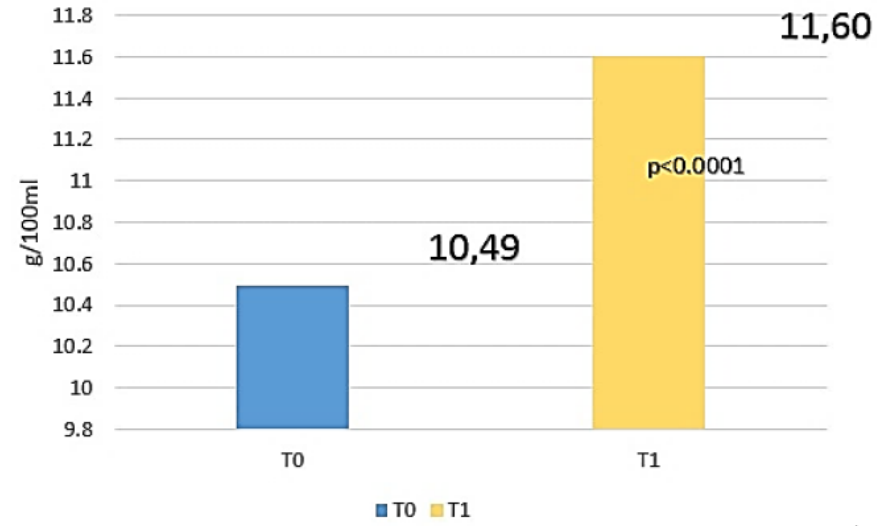

Figure 4. Repeated misures analysis of variance (ANOVA test) for Haemoglobin (HB) between 0 and $\mathrm{T} 1$ (2).

Co-processed compounds or microspheres with ALG could be prepared in several methods: single emulsion, solvent evaporation, cross-linking or spray-drying: recent published data on Ferrous Bysglicinate Chelate (co-processed ) (2-4) and on Ranitidine (microsphere) [12] have shown that spray drying techniques seems to be useful allowing to the final compound the best pharmacokinetic/ pharmakodinamic profile because of its particle size distribution, residual moisture content, bulk density and morphology (Figure 1) confirming the well known "dispert effect" of ALG also for IDA and ID treatment.

\section{References}

1. Alleyne M (2008) Individualized treatment for iron deficiency anemia in adults. $A m$ JMed 121: 943-948.

2. Ame A, Campa E (2016) FERALGINE® A New Oral Iron Therapy for Iron Deficiency Anemia: Preliminary Clinical Results on a Case Series of 12 Anemic Patients. Research \& Reviews: Pharmacy \& Pharmaceutical Sciences 5: 29-35.

3. Gervasi GB, Baldacci M, Bertini M (2016) Feralgine ${ }^{\circledR}$ a New Co-processed Substance to Improve Oral Iron Bioavailability, Taste and Tolerability in Iron Deficiency Patients. Archives of Medicine 8: 1- 13.

4. Rondinelli MB, Di Bartolomei A, De Rosa A, Pierelli L (2017) Oral Iron Absorption Test (OIAT): A Forgotten Screening Test for Iron Absorption from the Gastrointestinal Tract. A Case Series of 14 Iron Deficiency Anemia (IDA) Patients Treated with FERALGINE $^{\circledR}$. J Blood Dis Med 2: 1-15.

5. Tønnesen HH and Karlsen J (2002) Alginate in drug delivery systems. Drug Dev. Ind. Pharm 28: 621-630.

6. Sachan KN, Pushkar S, Jha A, Bhattcharya A (2009) Sodium alginate: the wonder polymer for controlled drug delivery. J. Pharm. Res 2: 1191-1199.

7. Ching AL, Liew CV, Chan LW, Heng PW (2008) Modifying matrix microenvironmental $\mathrm{pH}$ to achieve sustained drug release from highly laminating alginate matrices. Eur. J. Pharm. Sci 33: 361-370.

8. Zentilin P, Dulbecco P, Savarino E, Parodi A, Iiritano E, et al. (2005) An evaluation of the antireflux properties of sodium alginate by means of combined multichannel intraluminal impedance and pH-metry. Aliment. Pharmacol. Ther 21: 29-34.

9. Shadab MD, Singh GK, Ahuja A, Khar RK, Babota S, et al. (2012) Mucoadhesive microspheres as a controlled drug delivery system for gastroretention. Syst. Rev. Pharm 3: 4-14

10. Kumar A, Jha S, Rawal R, Chauhan PS, Maurya SD, et al. (2013) Mucoadhesive microspheres for novel drug delivery system: a review. Am. J. PharmTech Res $3: 196-213$.

11. Shadab M, Ahuja A, Khar RK, Baboota S, Chuttani K, et al. (2011) Gastroretentive drug delivery system of acyclovir-loaded alginate mucoadhesive microspheres: formulation and evaluation. Drug Deliv 18: 255-264.

12. Szelaska M, Amelian A, Winnicka K (2015) Alginate microspheres obtained by the spray drying technique as mucoadhesive carriers of ranitidine. Acta Pharm 65: 15-27. 
Baldacci M (2018) Iron deficiency anemia (ida) and iron deficiency (id): are alginates a good choise to improve oral iron bioavailability and safety?

13. Lee S, Kim YC, Park JH (2016) Zein-alginate based oral drug delivery systems: Protection and release of therapeutic proteins. Int J Pharm 515: 300-306.

14. Okunlola A, Odeku OA, Lamprecht A, Oyagbemi AA, Oridupa OA, et al. (2015) Design of cissus-alginate microbeads revealing mucoprotection properties in antiinflammatory therapy. Int J Biol Macromol 79: 226-34.
15. Opinion of the Scientific Panel on Food Additives, Flavourings, Processing Aids and materials in Contact with Food on a request from the Commission related to Ferrous bisglycinate as a source of iron for use in the manufacturing of foods and in food supplements. The EFSA Journal 299: 1-17.

Copyright: $(02018$ Baldacci M. This is an open-access article distributed under the terms of the Creative Commons Attribution License, which permits unrestricted use, distribution, and reproduction in any medium, provided the original author and source are credited. 